\title{
Microscopic Polyangiitis
}

\author{
Sharon A. Chung, MD, MAS and \\ Assistant Professor of Medicine, Rosalind Russell Medical Research Center for Arthritis, Division \\ of Rheumatology, University of California, San Francisco, CA, Sharon.Chung@ucsf.edu \\ Philip Seo, MD, MHS \\ Co-Director, the Johns Hopkins Vasculitis Center, Assistant Professor of Medicine, Johns \\ Hopkins University Division of Rheumatology, seo@jhmi.edu

\section{Synopsis} \\ In 1923, Friedrich Wohlwill described two patients with a "microscopic form of periarteritis \\ nodosa", which was distinct from classical polyarteritis nodosa. This disease, now known as \\ microscopic polyangiitis (MPA), is a primary systemic vasculitis characterized by inflammation of \\ the small-caliber blood vessels and the presence of circulating antineutrophil cytoplasmic \\ antibodies (ANCA). Typically, microscopic polyangiitis presents with glomerulonephritis and \\ pulmonary capillaritis, although involvement of the skin, nerves, and gastrointestinal tract is not \\ uncommon. Treatment of MPA generally requires use of a cytotoxic agent (such as \\ cyclophosphamide) in addition to high-dose glucocorticoids. Recent research has focused on \\ identifying alternate treatment strategies that minimize or eliminate exposure to cytotoxic agents. \\ This article will review the history, pathogenesis, clinical manifestations, and treatment of MPA.
}

Microscopic polyangiitis is an idiopathic autoimmune disease characterized by a systemic vasculitis that predominantly affects the small- caliber blood vessels, and is associated with the presence of antineutrophil cytoplasmic autoantibodies (ANCA). Because of its relationship to ANCA, it is often classified as a form of ANCA-associated vasculitis, an important subset of the primary systemic vasculitides that includes Wegener's granulomatosis (WG), the Churg-Strauss syndrome (CSS), and renal-limited vasculitis. Because it can lead to both pulmonary capillaritis and glomerulonephritis, MPA is also a prime cause of the pulmonary-renal syndrome, a group of disorders that includes Goodpasture's syndrome (which is associated with anti-glomerular basement membrane [GBM] antibodies), systemic lupus erythematosus, and WG. In this review, we will discuss the history, pathogenesis, clinical manifestations, and treatment of MPA.

\section{Historical Overview and Epidemiology}

Although syphilitic aneurysms had been recognized since the 1500s, the first complete description of a primary systemic vasculitis came in 1866, when Kussmaul and Maier described the plight of Carl Seufarth, a 27 year old journeyman tailor who had rapidly become incapacitated by fevers, myalgias, renal insufficiency, neuropathy, and abdominal

\section{(C) 2010 Elsevier Inc. All rights reserved.}

Corresponding Author: Philip Seo, MD, MHS, 5501 Hopkins Bayview Circle, JHAAC Room 1B.1A, Baltimore MD 21224, Telephone: 410-550-6813, Fax: 866-752-7405.

Publisher's Disclaimer: This is a PDF file of an unedited manuscript that has been accepted for publication. As a service to our customers we are providing this early version of the manuscript. The manuscript will undergo copyediting, typesetting, and review of the resulting proof before it is published in its final citable form. Please note that during the production process errors may be discovered which could affect the content, and all legal disclaimers that apply to the journal pertain. 
pain. At autopsy, they described "[p]eculiar mostly nodular thickening ... of countless arteries of and below the caliber of the liver artery and the major branches of the coronary arteries of the heart, principally in the bowel, stomach, kidneys, spleen, heart, and voluntary muscles, and to a lesser extent also in the liver, subcutaneous cell tissue and the bronchial and phrenic arteries."[1] Although the significance of these findings, which they dubbed "periarteritis nodosa", was not immediately clear, this is now widely recognized as the archetypal description of polyarteritis nodosa.[2]

For years after this description, all patients with a non-infectious arteritis were classified as having polyarteritis nodosa. In 1923, Friedrich Wohlwill described two patients who appeared to have a novel form of this disease, characterized by the presence of glomerulonephritis and non-granulomatous inflammation of the small-caliber blood vessels. [3] This "microscopic form of periarteritis nodosa" was gradually recognized as a new entity, distinct from classic polyarteritis nodosa. In 1953, Pearl Zeek noted that this disease was pathologically similar to hypersensitivity vasculitis, preferentially involving the arterioles and venules of the visceral organs (including the lung) but often sparing the medium-caliber blood vessels. [4] In 1950, Wainwright and Davson used the phrase "microscopic polyarteritis" to describe this phenotype.[5]

In 1985, Caroline Savage et al. defined "microscopic polyarteritis" as a small vessel vasculitis associated with focal segmental glomerulonephritis and hemoptysis.[6] In 1994, the Chapel Hill Consensus Conference proposed the term "microscopic polyangiitis" to describe patients with a small-vessel vasculitis characterized by the absence of immune complex deposition on immunofluorescence, and the presence of pulmonary capillaritis and glomerulonephritis.[7] The new name emphasized the differences between this phenomenon and "classic" polyarteritis nodosa, which was defined as a medium-vessel vasculitis that spared the arterioles and venules. Despite this clear distinction, distinguishing these two phenomena clinically is not always straightforward; the classic description of polyarteritis nodosa by Kussmaul and Maier, for example, includes evidence of a small vessel vasculitis. [8] Moreover, the Chapel Hill Consensus Conference criteria do not always clearly distinguish MPA from other forms of vasculitis, such as Wegener's granulomatosis.[9] Regardless, the introduction of this nomenclature resulted in a rapid reduction in the prevalence of polyarteritis nodosa, due to the reclassification of many of these patients as having MPA.[10]

In 1954, Godman and Churg noted that the "microscopic form of periarteritis" was closely related to WG and CSS.[11] In the ensuing years, it gradually became clear that these three forms of systemic vasculitis were also linked by the presence of anticytoplasmic antibodies directed against neutrophils. Antineutrophil cytoplasmic antibodies (ANCA) were first reported in association with focal segmental glomerulonephritis in the 1980s.[12] Subsequent work demonstrated that these antibodies were associated with distinct staining patterns when alcohol-fixed neutrophils were used as a substrate. In 1988, Jennette and Falk reported that serum from patients with WG, renal-limited vasculitis, and MPA was associated with antibodies that created a perinuclear staining pattern.[13] This p-ANCA pattern is caused by antibodies against myeloperoxidase. Some authors have suggested that MPO-ANCA be used to distinguish MPA from polyarteritis nodosa, [14] although these antibodies are also found in other forms of vasculitis, including drug-induced ANCAassociated vasculitis, CSS, and WG.

Regardless, ANCA has become a useful tool for the diagnosis of vasculitis, and may be partially responsible for the perceived increase in prevalence of the primary systemic vasculitides.[15] Southern Sweden has the highest reported prevalence of MPA, with 94 cases per million.[16] Overall, however, the incidence of MPA is higher in southern Europe 
than in northern Europe; for example, the incidence of MPA in Norway is 2.7 per million, [17] but 11.6 per million in Spain (see Table 1).[18] The incidence and prevalence of MPA in other parts of the world is less clear, but the prevalence seems to be higher in European populations.[19]

\section{Pathogenesis}

Growing evidence indicates that ANCA play a role in the pathogenesis of MPA. In theory, this might occur in two steps. In the first step, neutrophils are primed by exposure to lowlevels of proinflammatory cytokines, such as interleukin- 1 or tumor necrosis factor- $\alpha$.[20] This process leads to surface expression of myeloperoxidase, followed by adherence of neutrophils to the endothelial surface of blood vessels or glomeruli. In the second step, neutrophils are activated by interaction with MPO-ANCA, either through binding of its substrate[21] or interaction with neutrophil Fc receptors.[22]

Two animal models support a potential role for MPO-ANCA in the pathogenesis of MPA, $[23,24]$ demonstrating that MPO-ANCA are sufficient to induce pulmonary capillaritis and glomerulonephritis given the correct biologic milieu. Also in support of this role is a case report describing pulmonary hemorrhage and renal insufficiency newborn infant, presumably mediated by passage of MPO-ANCA from mother to fetus.[25] A subsequent case report, however, documents that placental transmission of MPO-ANCA is not sufficient to induce disease.[26] The development of vasculitis likely requires the presence of several co-factors, including genetic predisposition, in order for ANCA to be pathogenic.

This model fails to address the substantial number of patients who are ANCA-negative at the time of diagnosis.[27] It is interesting to note that not all patients with active vasculitis are ANCA positive, and MPO-ANCA titers themselves correlate poorly with disease activity in MPA. These observations imply that ANCA are not essential to pathogenesis in all patients with MPA, or that more than one mechanism can lead to the same clinical diagnosis. For example, recent work indicates that ANCA directed against lysosomal membrane protein-2 (LAMP-2), possibly induced by exposure to FimH-expressing gram negative bacteria, may play a key role in the development of vasculitis in some patients[28] (although this work has not yet been widely replicated).

\section{Clinical Features}

MPA has a slight male predominance (male:female ratio of 1.8:1) [14,29,30], with an average age of onset between 50-60 years [14,19,29]. As expected for an illness that affects multiple organ systems, patients with MPA can present with a myriad of different symptoms. However, over $70 \%$ of patients have constitutional symptoms such as fever or weight loss at diagnosis.[6,29] Patients can present acutely (i.e., having symptoms from days to weeks) or have an indolent course before diagnosis. For example, nonspecific symptoms such as a flu-like illness[6] or arthralgias can be present for months to years prior to diagnosis.[30] In this section, we will discuss the major clinical manifestations of MPA, presented by organ system.

\section{Renal manifestations}

Renal involvement, characterized by rapidly progressive glomerulonephritis (RPGN), is the major clinical feature of MPA. Previous studies report that $80-100 \%$ of patients with MPA experience renal manifestations $[6,14]$, which can range from an asymptomatic urinary sediment to end-stage renal disease requiring dialysis.[30] Consistent with glomerulonephritis, the most common clinical manifestations of renal involvement are 
proteinuria (in the nephrotic range in up to $50 \%$ of patients), microscopic hematuria, and urinary granular or red blood cell casts.[6]

The hallmark finding on renal biopsy is focal segmental necrotizing glomerulonephritis, which is seen in up to $100 \%$ of patients (Figure 1).[6] Glomerular crescents are also common, and can be present in approximately $90 \%$ of patients.[6] Frank vasculitis and fibrinoid necrosis are seen less frequently, and observed in less than $20 \%$ of patients.[6] Areas away from the glomeruli can be affected as well; for example, interstitial nephritis and tubular atrophy are seen in approximately half of MPA patients.[6] Immunofluorescence demonstrates minimal deposition of immunoglobulins or complement in the glomeruli and renal vessels (hence the descriptive term "pauci-immune"), unlike other small vessel vasculitis diseases such as Henoch-Schönlein purpura, cryoglobulinemic vasculitis, or antiGBM disease.[31] The changes seen on renal biopsy are similar for the three ANCAassociated vasculitidies (MPA, WG, and CSS), and thus cannot be used to distinguish between these entities.

The mainstay of treatment for renal involvement in MPA is glucocorticoids and cyclophosphamide. With such therapy, approximately $90 \%$ of patients enter a complete or partial remission.[32] However, despite immunosuppression, approximately $20 \%$ of patients in one series progressed to end stage renal disease and required renal replacement therapy, either in the form of kidney transplant or dialysis. Not surprisingly, a low serum creatinine at time of diagnosis in this group predicted a better renal survival rate.[32]

Of note, ANCA and anti-GBM antibodies can co-exist. Approximately $30 \%$ of patients with anti-GBM antibodies have circulating ANCA.[33,34] Conversely, a lower percentage (5$14 \%$ ) of patients with a positive ANCA have evidence of anti-GBM antibodies.[33,35] In patients with concurrent ANCA and anti-GBM antibodies, the majority of the ANCA are directed against MPO (66-100\%).[33-35] In a series of 22 patients with both antibodies who underwent renal biopsy, all biopsies showed linear deposition of IgG and C3 on immunofluorescence. Four of the 22 biopsies (18.2\%) also showed granular deposition of IgG, IgM, and C3. Patients with both ANCA and anti-GBM antibodies are sometimes treated with plasma exchange in addition to conventional immunouppression.[33] Studies of whether these "double positive" patients have worse renal outcomes compared to those with only ANCA or anti-GBM antibodies have conflicting results.[33] Some suggest that these patients are more likely to relapse than those patients with anti-GBM antibodies alone. $[34,36]$ Regardless, lower serum creatinine at diagnosis is associated with an increased renal survival rate.[33]

\section{Pulmonary manifestations}

Pulmonary involvement can be seen in $25-55 \%$ of patients. Manifestations include hemoptysis and alveolar hemorrhage, infiltrates, pleural effusion, pulmonary edema, pleuritis, and interstitial fibrosis. $[6,14,30]$ The classic pulmonary manifestation of MPA is diffuse alveolar hemorrhage caused by pulmonary capillaritis, which has been reported in 12-55\% of patients.[37-39] Common presenting symptoms of alveolar hemorrhage include dyspnea, cough, hemoptysis, and pleuritic chest pain.[40]

In patients with alveolar hemorrhage, chest radiographs show patchy, bilateral airspace opacities, usually involving both the upper and lower lung fields.[37,39] The most common finding on computed tomography (CT) is ground-glass attenuation (seen in $>90 \%$ of patients), which corresponds to alveolar hemorrhage, interstitial chronic inflammation of the alveolar septa, and capillaritis (see Figure 2). Consolidation is seen in $~ 80 \%$ of patients. Thickening of the bronchovascular bundles and honeycombing is also observed.[37,39] 
Patients often undergo bronchoscopy to evaluate the cause of bleeding. For patients with alveolar hemorrhage, bronchoalveolar lavage (BAL) fluid is usually grossly hemorrhagic, and on sequential lavage, the fluid remains bloody.[37,39,41] Perls Prussian blue staining of the BAL fluid shows elevated numbers of hemosiderin-laden macrophages (which are present in over $30 \%$ of patients with MPA).[37,39]

Lung biopsy of alveolar hemorrhage can show intra-alveolar and interstitial red blood cells, pauci-immune, hemorrhagic necrotizing alveolar capillaritis[38], neutrophilic infiltration resulting in fibrinoid necrosis and dissolution of the arterial and venular walls [41], and intra-alveolar hemosideroisis.[37] Of note, granulomatous inflammation is usually not observed in MPA and its presence suggests an alternative diagnosis, such as WG.

Pulmonary function testing can show either restrictive or obstructive patterns. The most frequent abnormality is reduced carbon monoxide diffusing capacity, which can increase dramatically during active alveolar hemorrhage.[38]

Treatment options for patients with alveolar hemorrhage due to MPA include aggressive immunosuppression and plasma exchange.[39] In severe cases, mechanical ventilation can be required to maintain oxygenation. A few case reports demonstrate successful use of extracorporeal membrane oxygenation.[39] However, alveolar hemorrhage is associated with a worse prognosis in patients with MPA. Patients with pulmonary hemorrhage are nine times more likely to die and have higher rates of relapse.[39]

Given the predominance of pulmonary and renal manifestations in MPA, MPA is a wellrecognized cause of pulmonary-renal syndromes, along with other autoimmune diseases such as WG, anti-GBM disease, and systemic lupus erythematosus. One study suggests that MPA is the most common cause of pulmonary-renal syndromes.[42]

Pulmonary fibrosis is a less well-recognized pulmonary manifestation of MPA.[43-45] Fibrosis can present months to years prior to, at time of, or years after the diagnosis of MPA. $[45,46]$ While the etiology of the fibrosis is unclear, chronic subclinical alveolar hemorrhage has been presented as a possible cause.[44] The prognosis for patients with pulmonary fibrosis is poor[45], but may be improved by the institution of immunosuppressive agents. $[38,44]$

More unusual pulmonary manifestations attributed to MPA include pulmonary artery aneurysms[47] and panbronchiolitis.[48]

\section{Skin manifestations}

Skin lesions are found in $30-60 \%$ of patients $[6,14,30,49]$, and are the initial presenting sign in 15-30\% of patients.[49] Palpable purpura is the most common manifestation, and occurs in $30-40 \%$ of patients.[49,50] Other manifestations include livedo reticularis, nodules, urticaria, and skin ulcers with necrosis.[49] Dermatologic manifestations have been associated with arthralgias in patients with MPA.[49]

Biopsies of palpable purpura often show leukocytoclastic vasculitis, with neutrophilic infiltration of the small-caliber vessels in the superficial dermis, fibrinoid necrosis, and nuclear dust.[50] However, a nonspecific perivascular lymphocytic infiltration can also be seen.[49,51] Biopsies of cutaneous nodules generally show vasculitis involving vessels of the deep dermis or subcutis.[49] Immunofluorescence studies are generally negative or show few deposits of immunoglobulins and complement.[49,51] While nodules are seen more frequently in polyarteritis nodosa, and palpable purpura is more frequent in MPA, both 
dermatologic manifestations occur in both diseases. Thus, these manifestations and their histologic findings cannot be used to differentiate between polyarteritis nodosa and MPA.

\section{Gastrointestinal manifestations}

The most frequently reported gastrointestinal symptom in MPA is abdominal pain[52], which can occur in 30-58\% of patients.[14,30] While gastrointestinal bleeding occurs in up to $21-29 \%$ of patients[52,53], massive hemorrhage is rare.[54] Angiographic studies, although not routinely performed, have shown arterial aneursyms [54,55] as a potential source of bleeding. Other gastrointestinal manifestations such as colonic ulcerations[56], intestinal ischemia[52,57], and bowel perforation[52] have been reported. However, they are likely less frequent in MPA compared to polyarteritis nodosa, since there are fewer reports of these manifestations in MPA patients in the published literature.

Involvement of the liver occurs rarely in MPA. Liver dysfunction in MPA may present as elevated liver enzymes, with alkaline phosphatase and $\gamma$-glutamyl transferase more affected than aspartate or alanine transaminase. These abnormal findings can precede the development of glomerulonephritis or pulmonary hemorrhage.[58-61] Histologic findings from liver biopsies performed from these cases have shown fibrinoid degeneration of an interlobular arteriole[60] as well as necrotizing arteritis and lymphocytic infiltration of portal tracts.[61] In addition, primary biliary cirrhosis has been reported in patients with MPA[62,63], but whether the association is causal is unknown.

\section{Neurologic manifestations}

Neurologic involvement in MPA is common, and affects between 37-72\% of patients. $[14,29,64]$ Peripheral neuropathy occurs more frequently than central nervous system involvement, with mononeuritis multiplex and distal symmetrical polyneuropathy as the predominant peripheral nervous system manifestations. Necrotizing vasculitis can be seen on sural nerve biopsy in up to $80 \%$ of affected patients, and nerve conduction studies typically show acute axonopathy.[65] Some studies suggest that relapse rates of peripheral neuropathy are low $[65,66]$, but this area warrants further investigation.

Central nervous system manifestations account for $17-30 \%$ of the neurologic involvement seen in MPA.[14,64] Manifestations are quite varied, and can include cerebral hemorrhage[29], pachymeningitis[67], and non-hemorrhagic cerebral infarctions.[68]

\section{Laboratory testing}

Currently, there is no laboratory test that has diagnostic specificity for MPA. Since ANCA are detected in only $50-75 \%$ of MPA patients[14,30], the absence of circulating ANCA does not exclude this diagnosis. ANCA associated with MPA generally has a perinuclear staining pattern (P-ANCA) caused by antibodies against myeloperoxidase (MPO-ANCA), which can be detected using enzyme-linked immunoassays (ELISA). Immunofluorescence has greater sensitivity, but the ELISA has greater specificity for the diagnosis of MPA. Unfortunately, neither test is specific for MPA, since these antibodies can be found in patients with the other ANCA-associated vasculitides in addition to other inflammatory diseases[69], such as drug-induced ANCA-associated vasculitis[70], cystic fibrosis[71], and various infections[72,73].

Nonspecific markers of inflammation are also observed in patients with MPA. The most common findings are an elevated erythrocyte sedimentation rate and C-reactive protein. Other findings include elevated white blood cell and platelet counts, and a normochromic, normocytic anemia.[53] 


\section{Treatment}

Given that most patients will present with glomerulonephritis, initial therapy of MPA generally entails the use of glucocorticoids and a cytotoxic agent such as cyclophosphamide. Using this regimen, remission can be achieved in $90 \%$ of patients. The regimen, first described by Anthony Fauci and Sheldon Wolfe for the treatment of WG, used daily oral cyclophosphamide ( $2 \mathrm{mg} / \mathrm{kg} / \mathrm{day})$ for years, both to induce and maintain remission.[74] Although effective, this regimen is associated with substantial toxicity, including infertility, malignancy, and hemorrhagic cystitis.[75] For this reason, substantial effort has been expended to find ways to minimize cyclophosphamide exposure, either by developing alternate dosing regimens or identifying subsets of patients who can be treated without resorting to cytotoxic agents.

\section{Remission maintenance strategies}

The Cyclophosphamide versus Azathioprine for early Remission phase of vasculitis trial (CYCAZAREM) treated 60 subjects with MPA and 95 subjects with WG who had involvement of the kidneys or another vital organ.[76] All subjects were treated with a remission induction regimen of daily oral cyclophosphamide $(2 \mathrm{mg} / \mathrm{kg} / \mathrm{day})$ and prednisolone for 3 to 6 months, after which subjects were randomized to receive either continued therapy with cyclophosphamide $(1.5 \mathrm{mg} / \mathrm{kg} /$ day $)$ or azathioprine $(2 \mathrm{mg} / \mathrm{kg} / \mathrm{day})$. Patients who had been randomized to receive cyclophosphamide were transitioned to azathioprine after having received 1 year of therapy. The primary endpoint of the trial was relapse rate, which was demonstrated to be equivalent among both groups (15\% versus $10 \%$, $\mathrm{P}=0.94)$.

CYCAZAREM effectively demonstrated that induction of remission with cyclophosphamide, followed by remission maintenance with azathioprine, was as effective at preventing disease flare as a prolonged course of cyclophosphamide. However, it was less clear if other remission maintenance agents, such as methotrexate, might be equally as effective. This question was addressed by the French Vasculitis Study Group, which treated 30 subjects with MPA and 96 subjects with WGs with intravenous cyclophosphamide (0.6 $\mathrm{mg} / \mathrm{m}^{2}$ every 2 weeks for 3 doses, then $0.6 \mathrm{mg} / \mathrm{m}^{2}$ every 3 weeks until remission was achieved, then an additional $0.7 \mathrm{mg} / \mathrm{m}^{2}$ every 3 weeks for 3 additional doses).[77] These subjects were subsequently randomized to receive remission maintenance therapy with either oral methotrexate (titrated to $25 \mathrm{mg} /$ week) or azathioprine $(2 \mathrm{mg} / \mathrm{kg} /$ day). The relapse rate was again determined to be equivalent in both groups ( $33 \%$ versus $36 \%, \mathrm{P}=0.71$ ).

Taken together, these trials indicate that for the treatment of MPA, remission induction with a limited course of cyclophosphamide, followed by remission maintenance with an antimetabolite such as methotrexate or azathioprine, is an appropriate treatment strategy for patients with severe disease. This leaves open the question, however, of whether adjunctive therapies, such as plasma exchange, might further augment the response to immunosuppression. This possibility was examined by the Methylprednisolone versus Plasma Exchange as additional therapy for severe ANCA associated glomerulonephritis trial (MEPEX) which enrolled 95 patients with MPA and 24 patients with WG, all of whom had biopsy-proven glomerulonephritis and a serum creatinine $\geq 5.8 \mathrm{mg} / \mathrm{dL}$.[78] All patients received treatment with oral glucocorticoids and cyclophosphamide $(2.5 \mathrm{mg} / \mathrm{kg} / \mathrm{day}$ for 3 months, then $1.5 \mathrm{mg} / \mathrm{kg} /$ day for an additional 3 months) followed by azathioprine ( $2 \mathrm{mg} / \mathrm{kg} /$ day). In addition, patients were randomized to receive adjunctive therapy with either intravenous methylprednisolone ( $1 \mathrm{~g}$ daily for 3 days) or a series of 7 plasma exchange procedures over 14 days. The primary endpoint of renal recovery at 3 months occurred more frequently among patients who had received plasma exchange (49\% versus $69 \%, \mathrm{P}=0.02$ ). Randomization to plasma exchange resulted in a $24 \%$ risk reduction of end-stage renal 
disease at 12 months (43\% versus 19\%). However, mortality was equivalent in both groups, and long-term follow-up studies demonstrate no difference in mortality or renal survival.

\section{Remission induction strategies}

Because of the toxicities inherent to the use of cyclophosphamide, investigators have focused on developing strategies to treat MPA that avoid using cytotoxic agents altogether. One such strategy involves identifying patients with milder disease, who may not require aggressive treatment to achieve remission. Silva et al. recruited 17 patients with MPA with mild to moderate renal involvement (defined as a serum creatinine $\leq 3 \mathrm{mg} / \mathrm{dL}$ ) for treatment with glucocorticoids and mycophenolate mofetil (2-3 g total dose daily).[79] Thirteen subjects $(76 \%)$ met the primary endpoint, and 12 remained in remission until month 18 . Regimens that avoid the use of cyclophosphamide for disease activity that does not affect life or the function of a vital organ may be a valuable strategy to help some patients avoid cytotoxic agents; research in analogous patients with WG indicates that methotrexate[80] and leflunomide[81] may also be effective for such patients.

Rituximab may represent an important alternative to cyclophosphamide for patients with higher levels of disease activity, which may not respond adequately to antimetabolite therapies. The Rituximab versus cyclophosphamide for induction of remission in ANCAassociated Vasculitis (RAVE) is a multi-center, double-blinded trial that randomized 48 subjects with MPA and 147 subjects with WG to receive either standard therapy or treatment with rituximab.[82] Standard therapy included a remission induction regimen of daily oral cyclophosphamide ( $2 \mathrm{mg} / \mathrm{kg} / \mathrm{day}$ ) for 3-6 months, followed by a remission maintenance regimen of azathioprine ( $2 \mathrm{mg} / \mathrm{kg} / \mathrm{day})$. Rituximab was administered using a standard lymphoma protocol $\left(375 \mathrm{mg} / \mathrm{m}^{2}\right.$ IV weekly for four weeks). This trial demonstrated that rituximab is non-inferior to cyclophosphamide for the induction of remission at 6 months (63.6\% versus $53.1 \%, \mathrm{P}=0.089)$. Remission rates at 6 months increase if subjects who were allowed to remain on low-dose glucocorticoids are included in the calculations (70.7\% versus $62.2 \%, \mathrm{P}=0.103$ ). Post hoc analysis indicates that rituximab may be especially effective for patients with relapsing disease (66.7\% versus $42.0 \%, \mathrm{P}=0.013$ ). Although the long-term consequences associated with rituximab treatment are not entirely clear for this patient population,[83] this trial strongly supports rituximab as an alternative to cyclophosphamide for the treatment of MPA.

\section{Summary}

MPA is a systemic necrotizing vasculitis with significant renal and pulmonary manifestations. The pathogenesis of MPA has not been clearly defined, although current evidence supports a role for ANCA. Diagnosis can be challenging, and relies on the physician drawing together elements of the patient's clinical history and symptoms with diagnostic tests such as tissue biopsy and autoantibody testing. Prognosis for MPA has greatly improved with the use of cyclophosphamide and glucocorticoids. The future of MPA treatment appears bright, as newer medications like rituximab show great promise as effective alternative therapeutic agents with potentially less toxicity.

\section{Acknowledgments}

Dr. Seo is a Lowe Family Scholar in the Johns Hopkins University Center for Innovative Medicine

Funding

The National Institutes of Health/National Institute of Arthritis and Musculoskeletal and Skin Diseases (1K23AR052820-01) 
American College of Rheumatology Physician Scientist Development Award National Institutes of Health/National Center for Research Resources (5 KL2 RR024130-04)

\section{References}

1. Matteson, E. Commemorative translation of the 130-year anniversary of the original article by Adolf Kussmaul and Rudolf Maier, ed. Anonymous. Rochester: Mayo Foundation; 1996.

2. Matteson EL. Historical perspective on the classification of vasculitis. Arthritis Care Res 2000;13(2):122-127. [PubMed: 14635285]

3. Wohlwill F. Über die nur mikroskopisch erkennbare Form der Periarteritis nodosa. Virchows Arch Pathol Anat Physiol 1923;246:36.

4. Zeek PM. Periarteritis nodosa and other forms of necrotizing angiitis. N Engl J Med 1953;248(18): 764-772. [PubMed: 13046612]

5. Wainwright J, Davson J. The renal appearances in the microscopic form of periarteritis nodosa. J Pathol Bacteriol 1950;62(2):189-196. [PubMed: 15437245]

6. Savage CO, et al. Microscopic polyarteritis: presentation, pathology and prognosis. Q J Med 1985;56(220):467-483. [PubMed: 4048389]

7. Jennette J, et al. Nomenclature of systemic vasculitides. Proposal of an international consensus conference. Arthritis Rheum 1994;37:187-192. [PubMed: 8129773]

8. Kussmaul A, Maier R. Ueber eine bisher nicht beschriebenen Eigenthumliche arterienerkrankung (periarteritis nodosa), die mit Morbus Brightii und rapid fortschreitender allgemeiner muskellahmung Einhergeht. Dtsch Arch Klin Med 1866;1:484-518.

9. Watts R, et al. Development and validation of a consensus methodology for the classification of the ANCA-associated vasculitides and polyarteritis nodosa for epidemiological studies. Ann Rheum Dis 2007;66(2):222-227. [PubMed: 16901958]

10. Watts RA, et al. Effect of classification on the incidence of polyarteritis nodosa and microscopic polyangiitis. Arthritis Rheum 1996;39(7):1208-1212. [PubMed: 8670332]

11. Godman G, Churg J. Wegener's granulomatosis: Pathology and review of the literature. Arch Pathol Lab Med 1954;58:533-553.

12. Davies D, et al. Segmental necrotising glomerulonephritis with antineutrophil antibody: possible arbovirus aetiology. BMJ 1982;285:606. [PubMed: 6297657]

13. Falk R, Jennette J. Anti-neutrophil cytoplasmic autoantibodies with specificity for myeloperoxidase in patients with systemic vasculitis and idiopathic necrotizing and crescentic glomerulonephritis. N Eng J Med 1988;318:1651.

14. Guillevin L, et al. Microscopic polyangiitis: clinical and laboratory findings in eighty-five patients. Arthritis Rheum 1999;42(3):421-430. [PubMed: 10088763]

15. Lane SE, et al. Primary renal vasculitis in Norfolk--increasing incidence or increasing recognition? Nephrol Dial Transplant 2000;15(1):23-27. [PubMed: 10607763]

16. Mohammad AJ, et al. Prevalence of Wegener's granulomatosis, microscopic polyangiitis, polyarteritis nodosa and Churg Strauss syndrome within a defined population in southern Sweden. Rheumatology (Oxford, England) 2007;46(8):1329-1337.

17. Koldingsnes W, Nossent H. Epidemiology of Wegener's granulomatosis in northern Norway. Arthritis Rheum 2000;43(11):2481-2487. [PubMed: 11083271]

18. Gonzalez-Gay MA, et al. The epidemiology of the primary systemic vasculitides in northwest Spain: implications of the Chapel Hill Consensus Conference definitions. Arthritis Rheum 2003;49(3):388-393. [PubMed: 12794795]

19. Mahr A, et al. Prevalences of polyarteritis nodosa, microscopic polyangiitis, Wegener's granulomatosis, and Churg-Strauss syndrome in a French urban multiethnic population in 2000: a capture-recapture estimate. Arthritis Rheum 2004;51(1):92-99. [PubMed: 14872461]

20. Kallenberg CG, Heeringa P, Stegeman CA. Mechanisms of Disease: pathogenesis and treatment of ANCA-associated vasculitides. Nature clinical practice.Rheumatology 2006;2(12):661-670.

21. Guilpain P, et al. Pathogenic effects of antimyeloperoxidase antibodies in patients with microscopic polyangiitis. Arthritis Rheum 2007;56(7):2455-2463. [PubMed: 17599755] 
22. Mulder A, et al. Activation of granulocytes by anti-neutrophil cytoplasmic antibodies (ANCA): a FcgRII-dependent process. Clin Exp Immunol 1994;98:270. [PubMed: 7955533]

23. Little MA, et al. Antineutrophil cytoplasm antibodies directed against myeloperoxidase augment leukocyte-microvascular interactions in vivo. Blood 2005;106(6):2050-2058. [PubMed: 15933057]

24. Xiao H, et al. Antineutrophil cytoplasmic autoantibodies specific for myeloperoxidase cause glomerulonephritis and vasculitis in mice. J Clin Invest 2002;110(7):955-963. [PubMed: 12370273]

25. Schlieben DJ, et al. Pulmonary-renal syndrome in a newborn with placental transmission of ANCAs. American Journal of Kidney Diseases : The Official Journal of the National Kidney Foundation 2005;45(4):758-761. [PubMed: 15806479]

26. Silva F, et al. Successful pregnancy and delivery of a healthy newborn despite transplacental transfer of antimyeloperoxidase antibodies from a mother with microscopic polyangiitis. Am J Kidney Dis 2009;54(3):542-545. [PubMed: 19395136]

27. Falk RJ, Hoffman GS. Controversies in small vessel vasculitis--comparing the rheumatology and nephrology views. Current opinion in rheumatology 2007;19(1):1-9. [PubMed: 17143089]

28. Kain R, et al. Molecular mimicry in pauci-immune focal necrotizing glomerulonephritis. Nat Med 2008;14(10):1088-1096. [PubMed: 18836458]

29. Agard C, et al. Microscopic polyangiitis and polyarteritis nodosa: how and when do they start? Arthritis Rheum 2003;49(5):709-715. [PubMed: 14558058]

30. Lhote F, Cohen P, Guillevin L. Polyarteritis nodosa, microscopic polyangiitis and Churg-Strauss syndrome. Lupus 1998;7(4):238-258. [PubMed: 9643314]

31. Jennette JC, Thomas DBS, Falk RJ. Microscopic polyangiitis (microscopic polyarteritis). Semin Diagn Pathol 2001;18(1):3-13. [PubMed: 11296991]

32. Westman KW, et al. Relapse rate, renal survival, and cancer morbidity in patients with Wegener's granulomatosis or microscopic polyangiitis with renal involvement. J Am Soc Nephrol 1998;9(5): 842-852. [PubMed: 9596082]

33. Levy JB, et al. Clinical features and outcome of patients with both ANCA and anti-GBM antibodies. Kidney Int 2004;66(4):1535-1540. [PubMed: 15458448]

34. Lindic J, et al. Clinical outcome of patients with coexistent antineutrophil cytoplasmic antibodies and antibodies against glomerular basement membrane. Ther Apher Dial 2009;13(4):278-281. [PubMed: 19695059]

35. Hellmark T, et al. Comparison of anti-GBM antibodies in sera with or without ANCA. J Am Soc Nephrol 1997;8(3):376-385. [PubMed: 9071706]

36. Lionaki S, Jennette JC, Falk RJ. Anti-neutrophil cytoplasmic (ANCA) and anti-glomerular basement membrane (GBM) autoantibodies in necrotizing and crescentic glomerulonephritis. Semin Immunopathol 2007;29(4):459-474. [PubMed: 17943286]

37. Lauque D, et al. Microscopic polyangiitis with alveolar hemorrhage. A study of 29 cases and review of the literature. Groupe d'Etudes et de Recherche sur les Maladies "Orphelines" Pulmonaires (GERM"O"P). Medicine (Baltimore) 2000;79(4):222-233. [PubMed: 10941351]

38. Gomez-Puerta JA, et al. Interstitial lung disease as a presenting manifestation of microscopic polyangiitis successfully treated with mycophenolate mofetil. Clin Exp Rheumatol 2009;27(1): 166-167. [PubMed: 19327249]

39. Collins CE, Quismorio FP Jr. Pulmonary involvement in microscopic polyangiitis. Curr Opin Pulm Med 2005;11(5):447-451. [PubMed: 16093820]

40. Franks TJ, Koss MN. Pulmonary capillaritis. Curr Opin Pulm Med 2000;6(5):430-435. [PubMed: 10958235]

41. Schwarz MI, Brown KK. Small vessel vasculitis of the lung. Thorax 2000;55(6):502-510. [PubMed: 10817800]

42. Niles JL, et al. The syndrome of lung hemorrhage and nephritis is usually an ANCA-associated condition. Arch Intern Med 1996;156(4):440-445. [PubMed: 8607730]

43. Eschun GM, Mink SN, Sharma S. Pulmonary interstitial fibrosis as a presenting manifestation in perinuclear antineutrophilic cytoplasmic antibody microscopic polyangiitis. Chest 2003;123(1): 297-301. [PubMed: 12527637] 
44. Birnbaum J, et al. Microscopic polyangiitis presenting as a "pulmonary-muscle" syndrome: is subclinical alveolar hemorrhage the mechanism of pulmonary fibrosis? Arthritis Rheum 2007;56(6):2065-2071. [PubMed: 17530647]

45. Tzelepis GE, et al. Prevalence and outcome of pulmonary fibrosis in microscopic polyangiitis. Eur Respir J. 2009

46. Foulon G, et al. ANCA-associated lung fibrosis: analysis of 17 patients. Respir Med 2008;102(10): 1392-1398. [PubMed: 18640019]

47. Ortiz-Santamaria V, et al. Pulmonary aneurysms in microscopic polyangiitis. Clin Rheumatol 2003;22(6):498-499. [PubMed: 14677042]

48. Park J, et al. Microscopic polyangiitis associated with diffuse panbronchiolitis. Intern Med 2004;43(4):331-335. [PubMed: 15168779]

49. Kluger N, et al. Comparison of cutaneous manifestations in systemic polyarteritis nodosa and microscopic polyangiitis. Br J Dermatol 2008;159(3):615-620. [PubMed: 18647311]

50. Kawakami T, et al. Cutaneous manifestations in patients with microscopic polyangiitis: two case reports and a minireview. Acta Derm Venereol 2006;86(2):144-147. [PubMed: 16648918]

51. Seishima M, Oyama Z, Oda M. Skin eruptions associated with microscopic polyangiitis. Eur J Dermatol 2004;14(4):255-258. [PubMed: 15319159]

52. Pagnoux C, et al. Presentation and outcome of gastrointestinal involvement in systemic necrotizing vasculitides: analysis of 62 patients with polyarteritis nodosa, microscopic polyangiitis, Wegener granulomatosis, Churg-Strauss syndrome, or rheumatoid arthritis-associated vasculitis. Medicine (Baltimore) 2005;84(2):115-128. [PubMed: 15758841]

53. Guillevin, L.; Pagnoux, C.; Teixeira, L. Microscopic polyangiitis, in Vasculitis. Ball, G.; Bridges, S., Jr, editors. Oxford: Oxford University Press; 2008. p. 355-364.

54. Ueda $\mathrm{S}$, et al. Microscopic polyangiitis complicated with massive intestinal bleeding. $\mathrm{J}$ Gastroenterol 2001;36(4):264-270. [PubMed: 11324731]

55. Spahn TW, et al. Gastrointestinal bleeding secondary to hepatic artery involvement of microscopic polyangiitis: case report and review of the literature. Dig Dis Sci 2007;52(6):1558-1561. [PubMed: 17410468]

56. Tsai CN, et al. Extended colonic ulcerations in a patient with microscopic polyangiitis. Ann Rheum Dis 2004;63(11):1521-1522. [PubMed: 15479909]

57. Passam FH, et al. Intestinal ischemia as the first manifestation of vasculitis. Semin Arthritis Rheum 2004;34(1):431-441. [PubMed: 15305242]

58. Ohnuma K, et al. Microscopic polyangiitis initiated with liver dysfunction, calf pain and fever of unknown origin. Rheumatol Int. 2009

59. Takebayashi K, et al. Microscopic polyangiitis presenting with liver dysfunction preceding rapidly progressive necrotizing glomerulonephritis. South Med J 2004;97(9):911-914. [PubMed: 15455987]

60. Nakamoto T, et al. Microscopic polyangiitis that presented liver dysfunction prior to noted renal manifestations. Intern Med 2000;39(6):517-521. [PubMed: 10852177]

61. Goritsas CP, et al. Intrahepatic bile duct injury and nodular regenerative hyperplasia of the liver in a patient with polyarteritis nodosa. J Hepatol 1997;26(3):727-730. [PubMed: 9075683]

62. Iannone F, et al. Microscopic polyangiitis associated with primary biliary cirrhosis. J Rheumatol 2003;30(12):2710-2722. [PubMed: 14719218]

63. Amezcua-Guerra LM, et al. Microscopic polyangiitis associated with primary biliary cirrhosis: a causal or casual association? J Rheumatol 2006;33(11):2351-2353. [PubMed: 16981285]

64. Zhang W, et al. Clinical analysis of nervous system involvement in ANCA-associated systemic vasculitides. Clin Exp Rheumatol 2009;27(1 Suppl 52):S65-S69. [PubMed: 19646349]

65 . Hattori N, et al. Mortality and morbidity in peripheral neuropathy associated Churg-Strauss syndrome and microscopic polyangiitis. J Rheumatol 2002;29(7):1408-1414. [PubMed: 12136898]

66. Cattaneo L, et al. Peripheral neuropathy in Wegener's granulomatosis, Churg-Strauss syndrome and microscopic polyangiitis. J Neurol Neurosurg Psychiatry 2007;78(10):1119-1123. [PubMed: 17299018] 
67. Furukawa Y, Matsumoto Y, Yamada M. Hypertrophic pachymeningitis as an initial and cardinal manifestation of microscopic polyangiitis. Neurology 2004;63(9):1722-1724. [PubMed: 15534267]

68. Ku BD, Shin HY. Multiple bilateral non-hemorrhagic cerebral infarctions associated with microscopic polyangiitis. Clin Neurol Neurosurg 2009;111(10):904-906. [PubMed: 19733002]

69. Stone J, et al. Test characteristics of Immunofluorescence and ELISA tests in 856 consecutive patients with possible ANCA-associated conditions. Arthritis Care and Research 2000;13(6):424434. [PubMed: 14635320]

70. Choi HK, et al. Drug-associated antineutrophil cytoplasmic antibody-positive vasculitis: prevalence among patients with high titers of antimyeloperoxidase antibodies. Arthritis Rheum 2000;43(2):405-413. [PubMed: 10693882]

71. Zhao MH, et al. Autoantibodies against bactericidal/permeability-increasing protein in patients with cystic fibrosis. Qjm 1996;89(4):259-265. [PubMed: 8733512]

72. Bauer A, et al. Vasculitic purpura with antineutrophil cytoplasmic antibody-positive acute renal failure in a patient with Streptococcus bovis case and Neisseria subflava bacteremia and subacute endocarditis. Clin Nephrol 2004;62(2):144-148. [PubMed: 15356972]

73. Hermann J, et al. Clinical interpretation of antineutrophil cytoplasmic antibodies: parvovirus B19 infection as a pitfall. Ann Rheum Dis 2005;64(4):641-643. [PubMed: 15485998]

74. Fauci A, Wolff S. Wegener's granulomatosis: studies in eighteen patients and a review of the literature. Medicine 1973;52:53-61. [PubMed: 4689172]

75. Hoffman GS, et al. Wegener's Granulomatosis: An Analysis of 158 Patients. Ann Intern Med 1992;116:488-498. [PubMed: 1739240]

76. Jayne D. Randomised trial of cyclophosphamide versus azathioprine during remission in ANCAassociated systemic vasculitis (CYCAZAREM). J Am Soc Nephrol 1999;10:105A.

77. Pagnoux C, et al. Azathioprine or methotrexate maintenance for ANCA-associated vasculitis. N Engl J Med 2008;359(26):2790-2803. [PubMed: 19109574]

78. Jayne DR, et al. Randomized trial of plasma exchange or high-dosage methylprednisolone as adjunctive therapy for severe renal vasculitis. J Am Soc Nephrol 2007;18(7):2180-2188. [PubMed: 17582159]

79. Silva F, et al. Mycophenolate Mofetil for Induction and Maintenance of Remission in Microscopic Polyangiitis with Mild to Moderate Renal Involvement--A Prospective, Open-Label Pilot Trial. Clin J Am Soc Nephrol.

80. De Groot K, et al. Randomized trial of cyclophosphamide versus methotrexate for induction of remission in early systemic antineutrophil cytoplasmic antibody-associated vasculitis. Arthritis and Rheumatism 2005;52(8):2461-2469. [PubMed: 16052573]

81. Metzler C, et al. Elevated relapse rate under oral methotrexate versus leflunomide for maintenance of remission in Wegener's granulomatosis. Rheumatology (Oxford, England) 2007;46(7):10871091.

82. J.H. Stone PAM, Seo P, Spiera R, Langford CA, Gary SHoffman, Kallenberg CGM, William St. Clair E, Fessler BJ, Tchao N, Ding L, Webber LV, Ikle D, Weitzenkamp D, Wu W, Brunetta P, Seismundo L, Fervenza FC, Keogh KA, Kissin EY, Mieras KS, Monach PA, Peikert T, Stegeman C, Ytterberg SR, Specks U. The RAVE-ITN Research Group. Rituximab Versus Cyclophosphamide for Induction of Remission in ANCA-Associated Vasculitis: A Randomized Controlled Trial (RAVE). Arthritis Rheum 2009;60 Supplement

83. Calabrese LH, Molloy ES. Therapy: rituximab and PML risk-informed decisions needed! Nat Rev Rheumatol 2009;5(10):528-529. [PubMed: 19798025]

84. Lane SE, Watts R, Scott DG. Epidemiology of systemic vasculitis. Curr Rheumatol Rep 2005;7(4): 270-275. [PubMed: 16045829] 


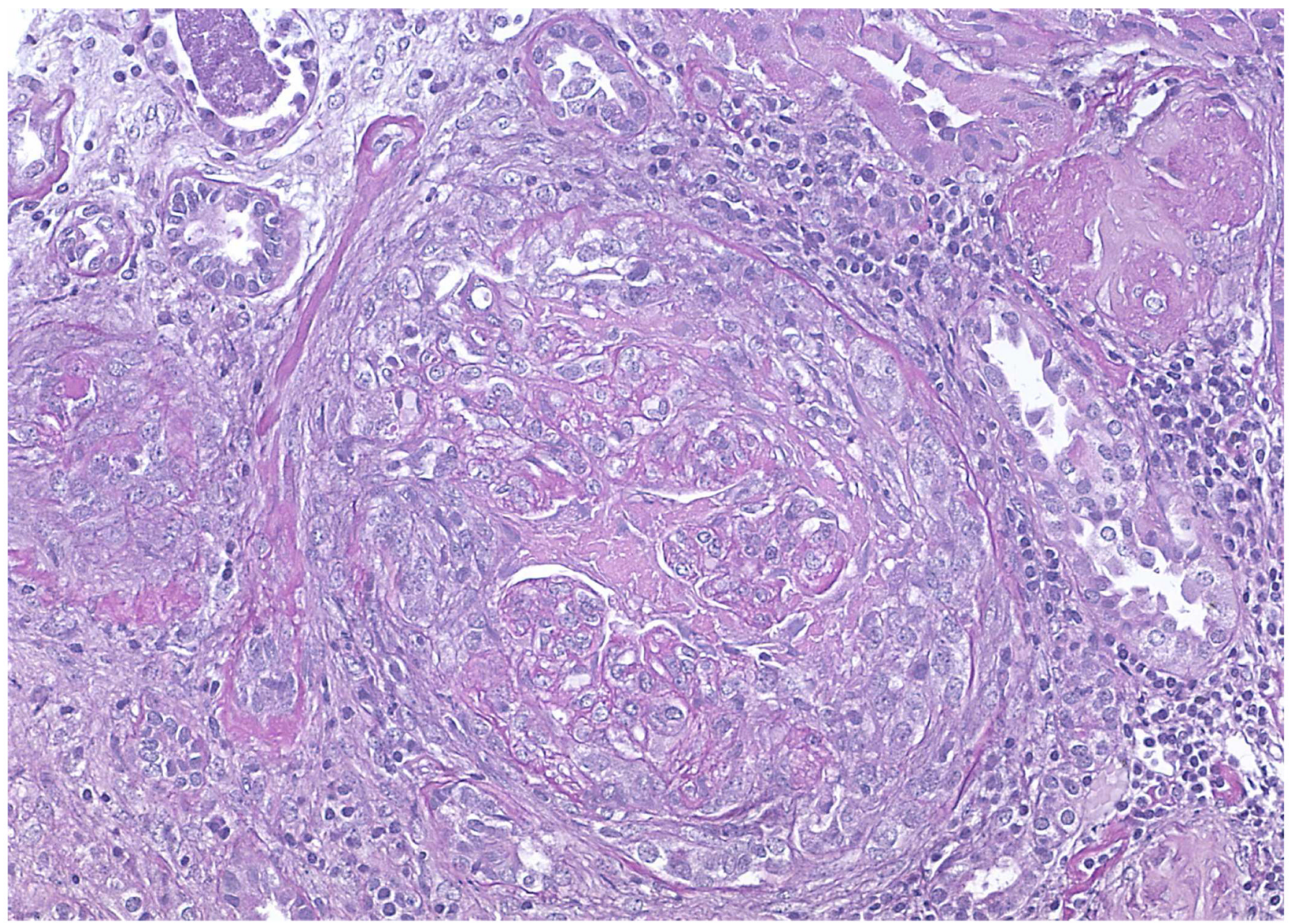

Figure 1.

Crescentic glomerulonephritis in a patient with microscopic polyangiitis (hematoxylin and eosin stain). 


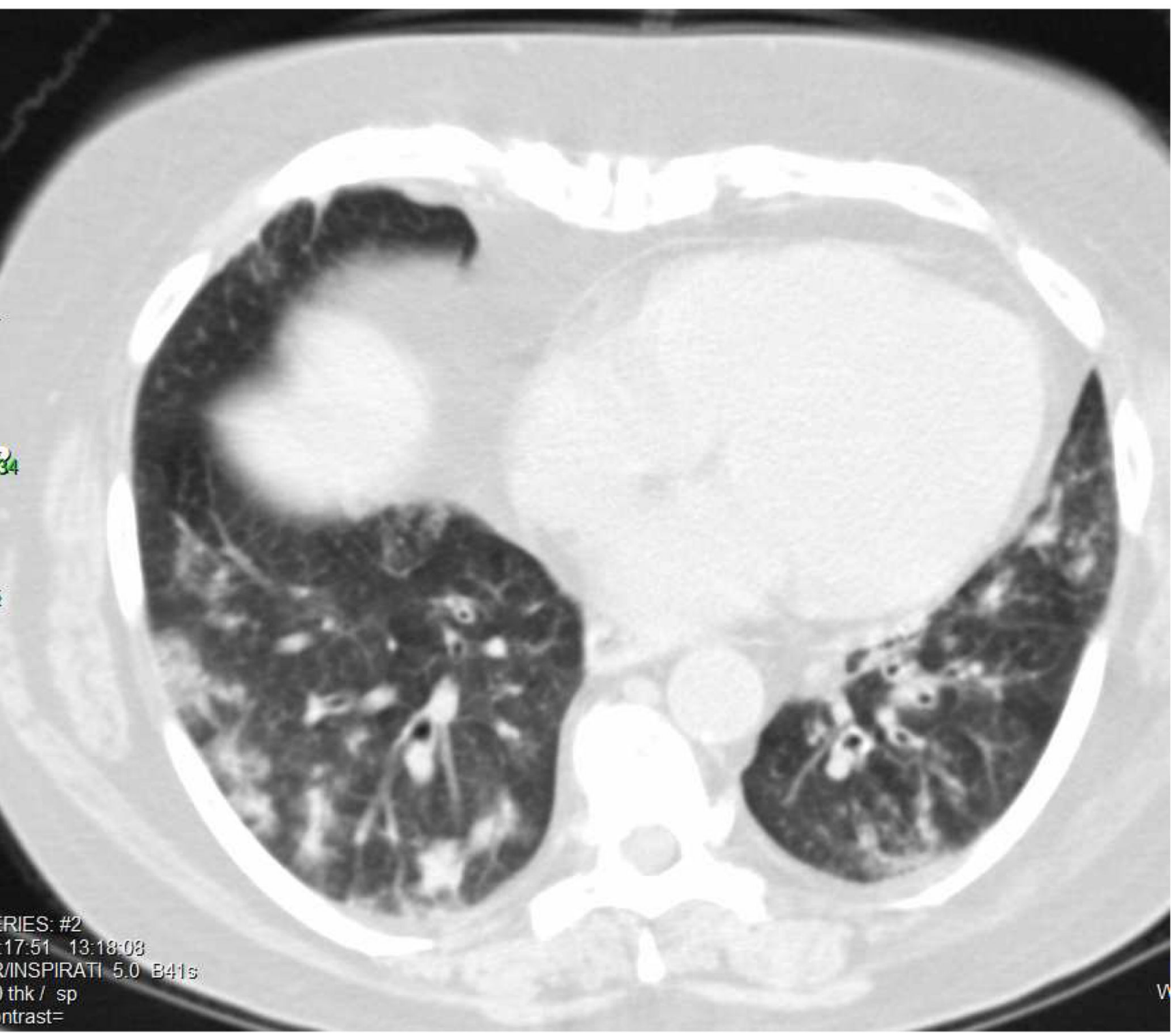

Figure 2.

Computed tomography scan demonstrating evidence of pulmonary hemorrhage in a patient with microscopic polyangiitis. 
Table 1

Incidence of microscopic polyangiitis in Europe

Data from reference [84]

\begin{tabular}{l|l}
\hline & Cases per million population \\
\hline Norway & 2.7 \\
\hline United Kingdom & 5.8 \\
\hline \begin{tabular}{l} 
Germany \\
\hline Spain
\end{tabular} & 2.6 \\
\hline
\end{tabular}

